\title{
ANATOMIA MICROCIRÚRGICA DO HIPOCAMPO NA AMÍGDALO-HIPOCAMPECTOMIA SELETIVA SOB A PERSPECTIVA DA TÉCNICA DE NIEMEYER E MÉTODO PRÉ-OPERATÓRIO PARA MAXIMIZAR A CORTICOTOMIA
}

\author{
Gustavo Rassier Isolan,2, Ney Azambuja', Eliseu Paglioli Neto', Eduardo Paglioli'
}

\begin{abstract}
RESUMO - O conhecimento da anatomia microcirúrgica do hipocampo tem importância fundamental na cirurgia da epilepsia do lobo temporal. Uma das técnicas mais utilizadas na cirurgia da epilepsia é a técnica de Niemeyer. Objetivo: Descrever em detalhes a anatomia do hipocampo e mostrar uma técnica na qual pontos de referências anatômicos pré-operatórios visualizados na RNM são usados para guiar a corticotomia. Método: Foram utilizados 20 hemisférios cerebrais e 8 cadáveres para dissecções anatômicas microcirúrgicas do lobo temporal e hipocampo para identificação e descrição das principais estruturas do hipocampo. Foram estudados prospectivamente 32 pacientes com epilepsia do lobo temporal refratários ao tratamento clínico submetidos a amígdalo-hipocampectomia seletiva pela técnica de Niemeyer três parâmetros anatômicos foram mensurados na RNM pré operatória e transferidos para o ato cirúrgico. Resultados: O hipocampo foi dividido em cabeça, corpo e cauda e sua anatomia microcirúrgica descrita em detalhes. As medidas adquiridas são apresentadas e discutidas. Conclusão: A complexa anatomia do hipocampo pode ser entendida de uma forma tridimensional durante dissecções microcirúrgicas. As medidas pré-operatórias mostraram-se guias anatômicos úteis para corticotomia na técnica de Niemeyer.
\end{abstract}

PALAVRAS-CHAVE: anatomia cirúrgica, hipocampo, amígdalo-hipocampectomia seletiva, cirurgia da epilepsia, vascularização.

\begin{abstract}
Hippocampal microsurgical anatomy regarding the selective amygdalohippocampectomy in the Niemeyer's technique perspective and preoperative method to maximize the corticotomy

ABSTRACT - The deep knowledge of hippocampal microsurgical anatomy is paramount in epilepsy surgery. One of the most used techniques is those proposed by Niemeyer. Purpose: To describe the hippocampal anatomy in details and to present a technique which preoperative anatomical points in MRI are identified to guide the corticotomy. Method: Microsurgical dissections were performed in twenty brain hemispheres and eight cadaveric heads to identify temporal lobe and hippocampus structures. Thirty two patients with drug-resistent temporal lobe epilepsy underwent a selective amygdalohippocampectomy with Niemeyer's technique being measured three preoperative MRI preoperative distances to guide the corticotomy. Results: The hippocampus was divided in head, body and tail and its microsurgical anatomy described in details. The MRI measurements are presented and discussed. Conclusion: The knowledge of the complex anatomy of the hippocampus can be achieved in a three-dimensional way during microsurgical dissections not. The preoperative MRI measurement is a reasonable guide to perform temporal corticotomy in Niemeyer's techinique.
\end{abstract}

KEY WORDS: surgical anatomy, hippocampus, selective amygdalohippocampectomy, epilepsy surgery, vascularization.

A anatomia cirúrgica do lobo temporal, especialmente de sua porção mesial, é complexa, exigindo um apurado conhecimento da equipe cirúrgica, sem o qual os resultados do tratamento serão prejudicados por altos índices de morbidade e escassos benefí- cios terapêuticos'. A amígdala, juntamente com o hipocampo, constitui uma das principais regiões do sistema límbico e é formada por duas porções maiores: a temporal ou principal, localizada no lobo temporal, e a extra temporal que se localiza no assoalho do ven-

Hospital São Lucas da Pontifícia Universidade Católica do Rio Grande do Sul, Departamento de Neurocirurgia, Programa da Cirurgia da Epilepsia, Porto Alegre RS, Brasil: 'Neurocirurgião; ${ }^{2}$ Fellowship observer.

Recebido 20 Março 2007, recebido na forma final 25 Junho 2007. Aceito 13 Agosto 2007.

Dr. Gustavo Rassier Isolan - Rua Ramiro Barcelos 2171 / 31. 90035-007 Porto Alegre RS - Brasil. E-mail: gisolan@yahoo.com.br 
trículo lateral ${ }^{1-3}$. A amígdala temporal em seu limite dorsal é contígua ao trato óptico e une-se ao globo pálido sem nenhum plano de clivagem. Ventralmente, a amígdala temporal projeta-se no interior do corno temporal em direção a cabeça do hipocampo, com o qual mantém íntima relação. Medialmente a amígdala temporal relaciona-se com o úncus. O recesso uncal, extensão anterior da eminência colateral, separa a amígdala da cabeça do hipocampo e com ela forma o limite anterior do corno temporal ${ }^{1-3}$.

O hipocampo é uma estrutura complexa que ocupa a porção medial do assoalho do corno temporal, formando um arco ao redor do mesencéfalo. Anatomicamente, pode ser dividido em cabeça, corpo e cauda. O hipocampo, que está localizado na base do lobo temporal, limita-se com a cisterna ambiens, continuando-se pelo úncus medialmente. Inferiormente repousa sobre o giro para-hipocampal e lateralmente relaciona-se com a fissura colateral e com o giro fusiforme. Denomina-se formação hipocampal o subículo, o hipocampo (cornu ammonis), citoarquiteturalmente dividido em quatro setores, e o giro denteado. O para-hipocampo repousa sobre as cisternas subaracnóideas da base do encéfalo e sobre o tentório.

Estima-se que entre 50 a $75 \%$ dos pacientes submetidos à cirurgia para epilepsia sejam para o tratamento da epilepsia refratária do lobo temporal, em que é indicada a ressecção cirúrgica da amígdala e da formação hipocampal na maioria dos casos ${ }^{4}$. Uma das técnicas mais utilizadas para ressecar estas estruturas é a amígdalo-hipocampectomia seletiva pela técnica de Niemeyer, na qual se realiza uma incisão de $2 \mathrm{~cm}$ em T2 (giro temporal médio) que é aprofundada em ângulo reto com o córtex cerebral até alcançar o corno temporal do ventrículo lateral, onde a amígdala é removida anteriormente e o hipocampo removido em bloco em seus 2 a 2,5 cm anteriores ${ }^{5}$. No que concerne às medidas anatômicas utilizadas na técnica de Niemeyer, estas não levam em consideração as variações que podem ser encontradas na anatomia causadas, em geral, pela patologia que afeta as estruturas temporais mesiais. Desse modo, medidas anatômicas, visando maximizar a exposição das estruturas a serem ressecadas poderiam ser aferidas individualmente na ressonância nuclear magnética (RNM) pré-opertória e transpostas para o campo cirúrgico, maximizando a corticotomia temporal.

Os objetivos deste estudo são descrever a anatomia microcirúrgica do hipocampo e apresentar os resultados da amígdalo-hipocampectomia seletiva pela técnica de Niemeyer de uma série de pacientes, estudados prospectivamente, nos quais medidas anatômicas pré estabelecidas na RNM de encéfalo foram usadas como referência no transoperatório.

\section{MÉTODO}

A parte anatômica deste estudo foi realizada por um dos autores (GRI) em dois diferentes laboratórios de microcirurgia. A primeira parte foi realizada no Laboratório de Microcirurgia do Hospital Beneficência Portuguesa de São Paulo e Instituto de Ciências Neurológicas, onde foram realizadas dissecções microcirúrgicas com utilização de bancada e microscópio cirúrgico com magnificação de três a 40 vezes e instrumental microcirúrgico em dez cérebros fixados em formalina com artérias e veias injetadas com material siliconado colorido num total de 20 hemisférios cerebrais, após ter sido aprovado pelo comitê de ética (HBP - protocolo 250/5). Utilizou-se silicone (Dow Corning 3110 RTV silicone rubber encapsulan, Midland, MI, USA) com corante vermelho para perfundir as artérias e com corante azul para perfusão venosa.

A segunda parte anatômica foi realizada no Dianne and M. Gazi Yasargil Education Center, Microsurgical Laboratory, na University of Arkansas for Medical Sciences, onde dissecções anatômicas foram realizadas em oito cadáveres fixados em Carolina's perfect solution ${ }^{\circledR}$ (Carolina Biological Supply Company, Burlington, NC, USA) com microscópio (Ziess) e material microcirúrgico.

Foram estudados prospectivamente 32 pacientes com epilepsia do lobo temporal refratários ao tratamento clínico que foram submetidos a amígdalo-hipocampectomia seletiva pela técnica de Niemeyer por um dos autores (N.A.), onde os seguintes parâmetros anatômicos foram mensurados na RNM pré operatória e transferidos para o ato cirúrgico: 1. distância vertical da margem inferior do sulco lateral (também conhecido como fissura silviana) à superfície da cabeça do hipocampo no corte coronal (Linha A), 2. distância horizontal do córtex temporal ao início do corno temporal do ventrículo lateral no corte coronal (Linha B), e 3 . distância da asa menor do osso esfenóide ao limite anterior do corno temporal do ventrículo lateral no corte axial (Linha C). O desenho dessas linhas na RNM pode ser visualizado na Figura 1. Todos os pacientes foram estudados com RNM em T1, T2 e inversion recovery.

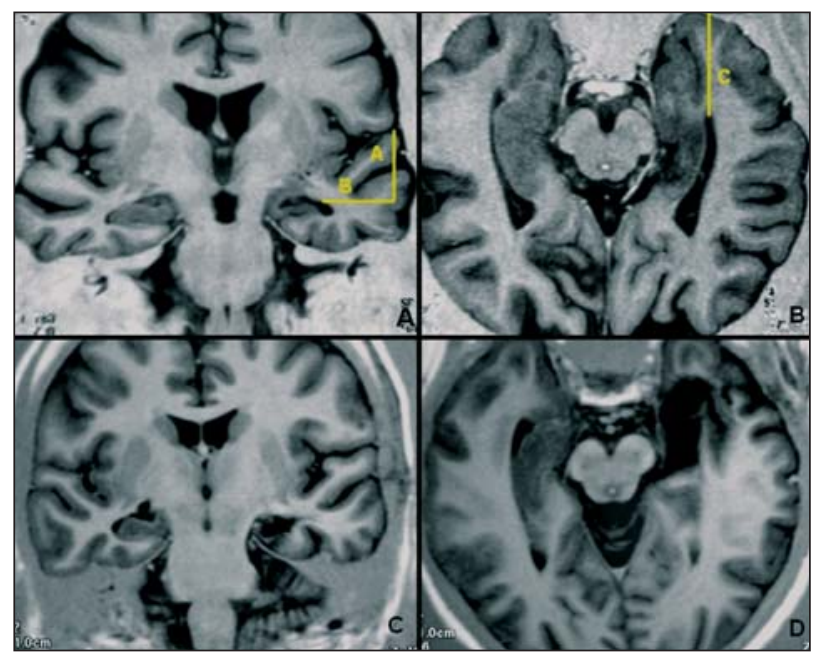

Fig 1. Ressonância Nuclear Magnética em T2 inversion recovery. A e B. Parâmetros medidos que são transpostos para a cirurgia. $C$ e D. Pós-operatório de amigdalohipocampectomia seletiva pela técnica de Niemeyer em cortes coronal e axial, respectivamente. 


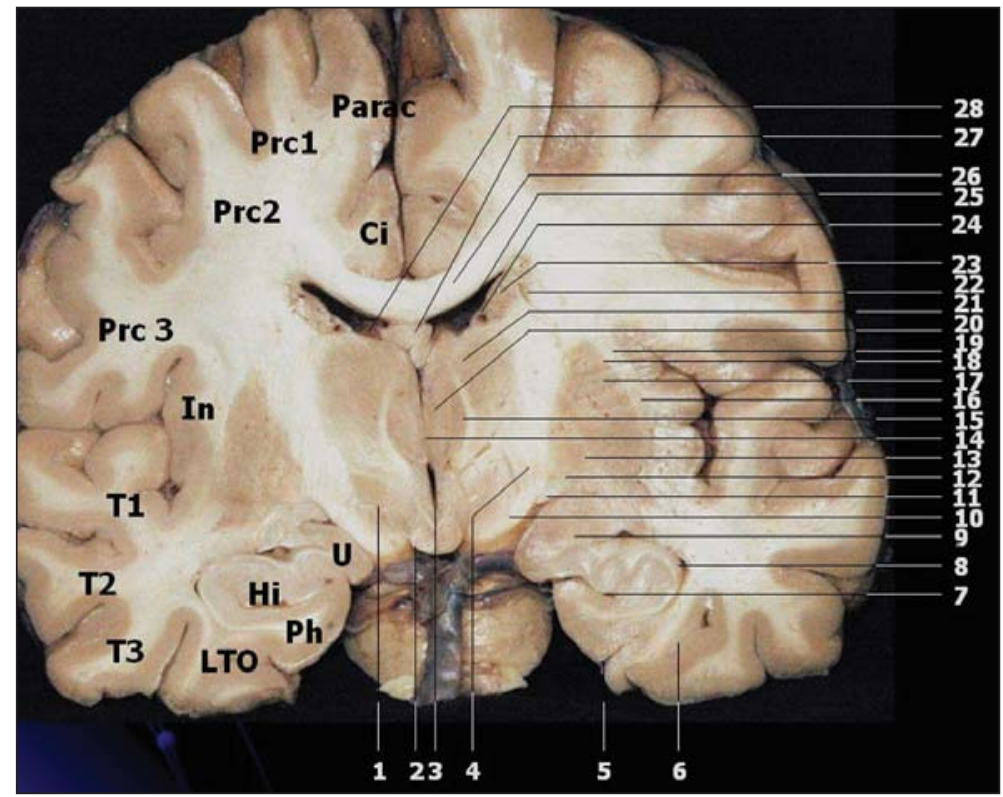

Fig 2. Corte coronal ao nível dos corpos mamilares e núcleos anteriores do tálamo 1) fascículo lenticular, 2) Corpo mamilar, 3) Terceiro ventrículo, 4) perna posterior da cápsula interna, 5) sulco colateral, 6) sulco occiptotemporal, 7) fissura hipocampal, 8) corno temporal do ventrículo lateral, 9) amígdala, 10) pedúnculo cerebral, 11) trato óptico, 12) globo pálido medial, 13) lâmina medular interna, 14) massa intermedia, 15) trato mamilotalâmico, 16) claustrum, 17) putamen, 18) cápsula externa,19) cápsula extrema, 20) núcleos anteriores do tálamo, 21) núcleos ventrais anteriores do tálamo, 22) estria cinzenta caudato-lenticular transcapsular, 23) núcleo caudado, 24) estria terminal e veia talamoestriada, 25) corpo do ventrículo lateral, 26) corpo caloso, 27) fornix, 28) plexo coróide, U) úncus, Hi) hipocampo, Ph) parahipocampo, LTO) giro occipito-temporal lateral, T1) giro temporal superior, T2) giro temporal médio, T3) giro temporal inferior, In) ínsula, Prc 1) pedúnculo superior do giro pré-central, Prc 2) pedúnculo médio do giro pré-central, Prc 3) pedúnculo inferior do giro pré-central, Parac) giro paracentral, Ci) giro do cíngulo.

\section{RESULTADOS}

Anatomia do hipocampo - A complexa anatomia do hipocampo pode ser descrita separadamente em cabeça, corpo e cauda, sendo cada uma destas regiões divididas em partes intraventricular e extraventricular, conforme descrito por Duvernoy4. As relações das estruturas cerebrais com o hipocampo em um corte coronal estão demonstradas na Figura 2.

Cabeça do hipocampo - Na parte intraventricular indentificam-se três a quatro digitações sagitalmente orientadas (digitações do hipocampo). Uma digitação vertical que corresponde à superfície medial do úncus pode ser identificada algumas vezes. O giro denteado cobre parte destas digitações. Da fímbria do hipocampo origina-se uma estrutura chamada alveus, que cobre as digitações do hipocampo ao nível da junção do corpo com a cabeça do deste. Uma particularidade anatômica é que sobre a cabeça do hipocampo não há plexo coróide. O recesso uncal é um prolongamento do corno temporal anterior ao hipocampo que se estende na porção mais profunda do úncus ${ }^{6}$. Os núcleos basais e laterais da amígdala são praticamente fusionados com a cabeça do hipocampo.

A parte extraventricular é composta pelos segmentos anterior e posterior do úncus. O segmento anterior do úncus cobre a amígdala e é formado pelos giros semilunar e ambiens, os quais são separados entre si pelo sulco semilunar. O segmento posterior do úncus forma parte do hipocampo e do subiculum e tem uma superfície inferior e uma medial. A superfície inferior está na profundidade do sulco uncal e pode ser visualizada após ressecção do giro para-hipocampal subjacen- te. Ela é dividida em banda de Giacomini, digitações externas e superfície inferior do ápex uncal. A banda de Giacomini é o segmento do giro denteado ao nível da cabeça do hipocampo. As digitações externas são dois ou três pequenos lóbulos anteriores à banda de Giacomini que representam "imagens invertidas" das digitações do hipocampo. A superfície inferior do ápex uncal é separada da banda de Giacomini posteriormente por um pequeno sulco. O ápex do úncus é formado por $\mathrm{CA}_{3}$ e CA 4 encobertos pelo alveus e forma o limite posterior do úncus. A superfície medial, por sua vez, é dividida pelo segmento terminal da banda de Giacomini, que está localizado no lábio superior do sulco uncal. Fazem parte a superfície medial do ápex uncal e o giro uncinato, o qual se une com o giro ambiens e é anterior à banda de Giacomini (Figuras 3 e 4).

Corpo do hipocampo - A parte intraventricular do corpo do hipocampo localiza-se no assoalho do corno temporal do ventrículo lateral. Ela é delimitada lateralmente pela eminência colateral, que corresponde na base do cérebro ao sulco colateral, e medialmente pela fímbria. O corpo do hipocampo é coberto por plexo coróide, que está aderido a uma dupla camada de tecido cerebral formada por pia-máter e epêndima, constituindo a tela coróide. O ponto coroideo inferior é um espaço triangular na superfície superior do úncus, onde a tênia da fímbria e a estria terminal se unem. A parte extraventricular é formada pelo giro denteado, fímbria e sulco hipocampal superficial.

Cauda do hipocampo - A parte intraventricular da cauda do hipocampo é delimitada medialmente 


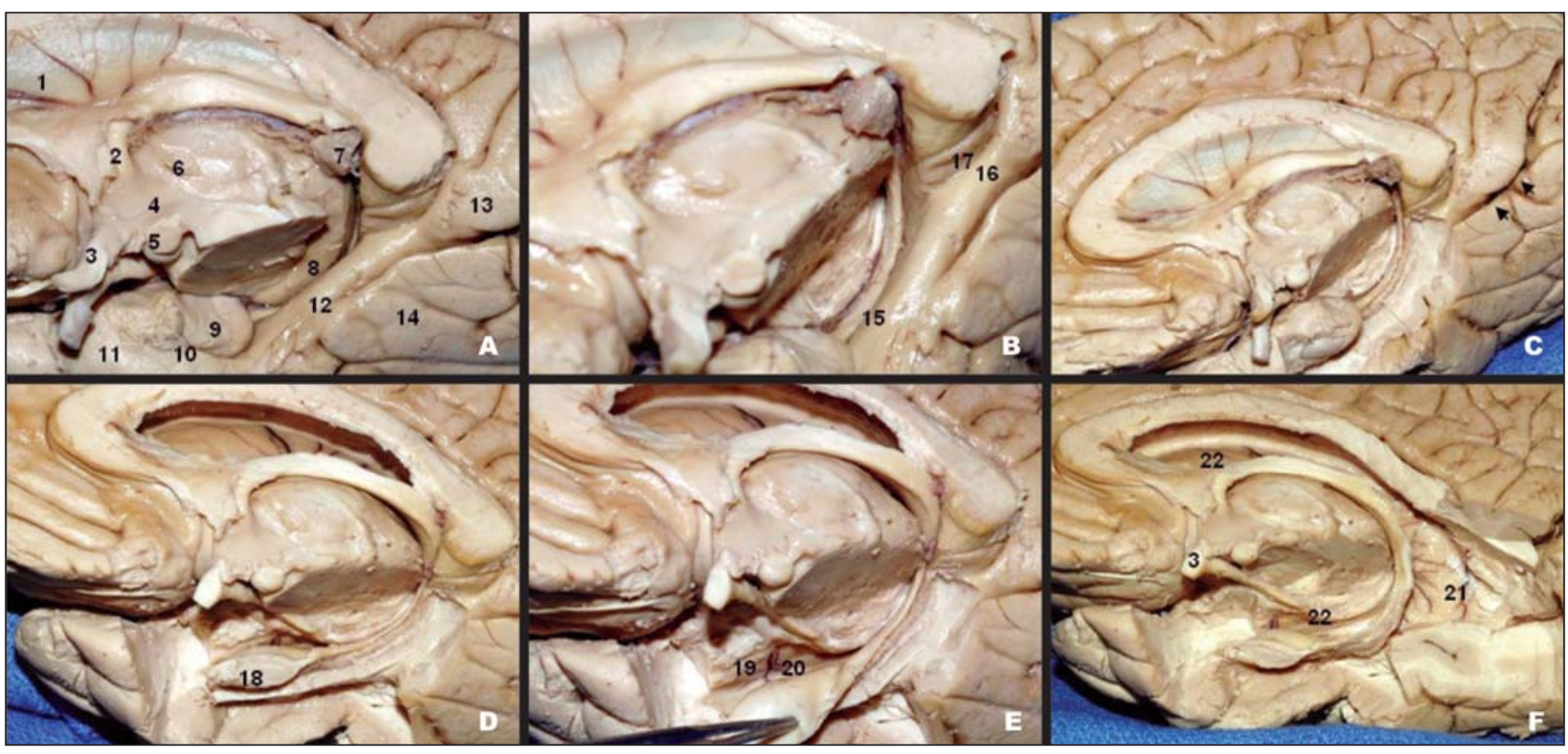

Fig 3. Visão medial do hemisfério cerebral direito. 1) septo pelúcido, 2) fórnix (coluna anterior), 3) quiasma óptico, 4) hipocampo, 5) corpo mamilar, 6) aderência intertalâmica, 7) glândula pineal, 8) pulvinar do tálamo, 9) úncus, 10) sulco ou incisura uncal, 11) pólo temporal, 12) giro para-hipocampal, 13) cíngulo, 14) giro lingual, 15) sulco hipocampal, 16) istmo, 17) giro fasciolar, 18) cabeça do hipocampo, 19) amígdala, 20) ponto coroideo inferior, 21) átrio ventricular, 22) trato óptico, 23) cabeça do núcleo caudado, 24) comissura anterior. Ponta de setas: sulco calcarino anterior.

e lateralmente pela fímbria e trígono colateral, respectivamente. O plexo coróide é maior ao nível desta região, sendo denominado glomo. Posteriormente o corpo do hipocampo atinge uma protrusão intraventricular chamada calcar avis. A parte extraventricular da cauda do hipocampo é dividida em segmentos inicial, médio e terminal. O segmento inicial forma o margo denticularis. $\mathrm{O}$ giro dentato possui várias extensões que penetram profundamente no hipocampo. No segmento médio o margo denticularis tornase estreito e liso, formando a fascíola cinérea. Ainda no segmento médio, a fímbria ascende para se unir à crura do fórnix e é possível identificar o giro fasciolar (CA3 coberto pelo alveus), o qual é separado da fascíola cinérea pelo sulco dentofascicular.

Vascularização do hipocampo - As cisternas da base, cisterna crural e ambiens abrigam em seu interior as estruturas vasculares e nervosas que devem ser preservadas no decorrer do procedimento cirúrgico ${ }^{7}$.

A cisterna crural, situada medialmente ao úncus e lateralmente ao pedúnculo cerebral, abriga a artéria coroidéia anterior, comunicando-se em sua porção posterior com a cisterna ambiens; esta circunda o mesencéfalo sendo limitada lateralmente pelo para-hipocampo contendo os seguimentos proximais da artéria cerebral posterior, os vasos coroideos posteriores e a porção mesencefálica da veia basal.

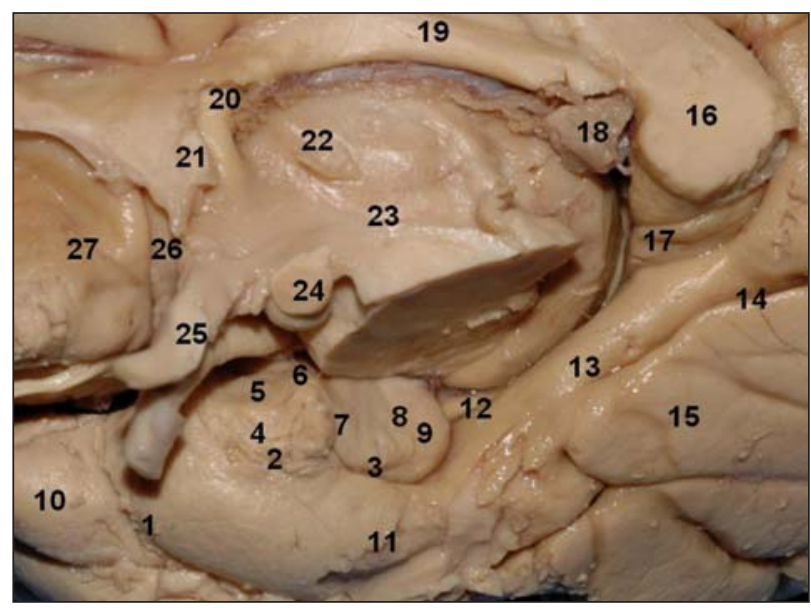

Fig 4. Aspecto medial do hemisfério cerebral direito. 1) sulco rinal, 2) incisura uncal, 3) sulco uncal, 4) giro ambiens, 5) giro semilunar, 6) sulco endorina, 7) giro uncinato, 8) banda de Giacomini, 9) giro intralimbic, 10) pólo temporal, 11) giro parahipocampal, 12) fimbria, 13) istmo, 14) sulco calcarino anterior, 15) giro lingual, 16) splenio do corpo caloso, 17) giro fasciolar, 18) glândula pineal , 19) corpo do fórnix, 20) coluna anterior do fornix, 21) comissura anterior, 22) aderência intertalâmica, 23) sulco hipotalâmico, 24) corpo mamilar, 25) quiasma óptico, 26) giro paraterminal, 27) giro subcaloso.

As relações vasculares do hipocampo com demais estruturas anatômicas podem ser visualizadas na Figura 5 , enquanto as relações das artérias intra-hipocampais na Figura 6. 


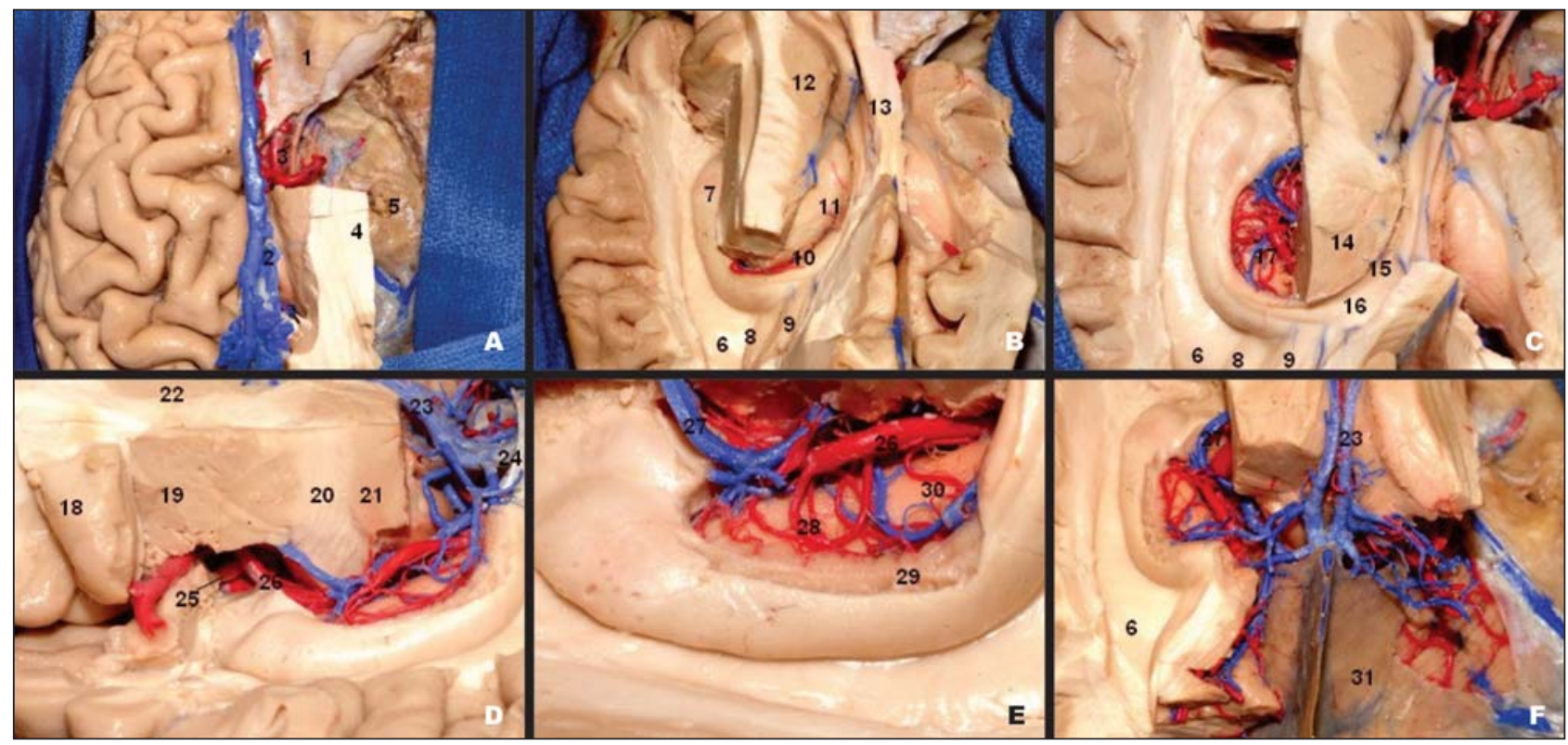

Fig 5. Relações anatômicas do hipocampo. Perspectivas superior ( $A, B, C, F)$ e lateral ( $D, E)$. 1) teto da órbita, 2) seio sagital superior, 3) artéria carótida interna, 4) cápsula interna, 5) assoalho da fossa média, 6) trígono colateral, 7) hipocampo, 8) calcar avis, 9) bulbo do corpo caloso, 10) fissura coroidéia, 11) tálamo, 12) cabeça do núcleo caudado, 13) fórnix, 14) pulvinar do tálamo, 15) parte atrial da fissure coroidéia, 16) crura do fórnix, 17) foramen interventricular (Monro), 18) giro curto da ínsula, 19) globus pálido, 20) cápsula interna, 21) pulvinar do tálamo, 22) núcleo caudado, 23) veia cerebral interna, 24) veia de Galeno, 25) nervo oculomotor, 26) artéria cerebral posterior, 27) veia basal (Rosenthal), 28) artéria hipocampal média, 29) giro denteado, 30) artéria hipocampal posterior; 31) tenda do cerebelo.

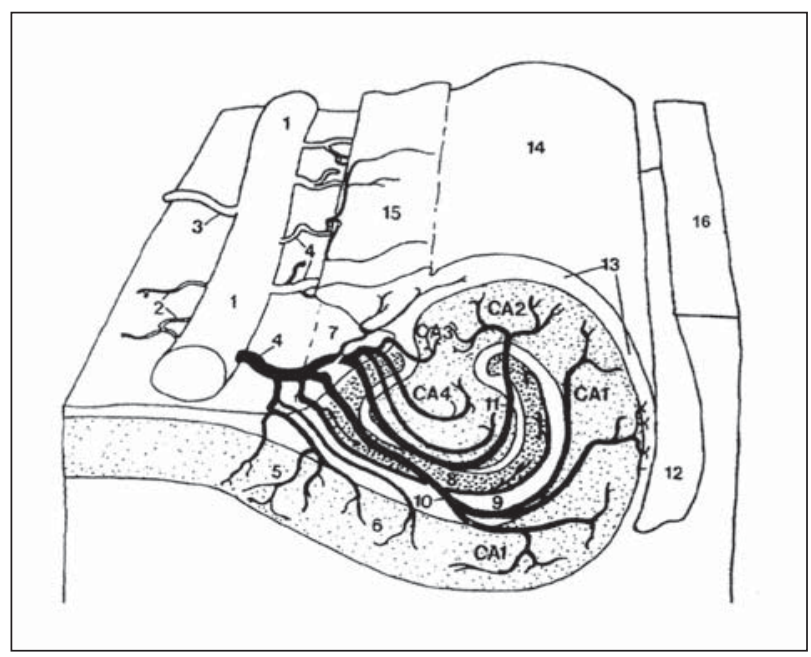

Fig 6. Vasos intra-hipocampais: corte coronal da porção média do hipocampo esquerdo. 1) tronco da artéria hipocampal média, 2) ramos para o subículo, 3) ramos para o para-hipocampo, 4) artérias retas, 5) subículo próprio, 6) prosubículo, 7) sulco fimbrio-denteado, 8) giro denteado, 9) lâmina medular entre o giro denteado e o hipocampo, 10) artéria intra-hipocampal ventral, 11) artéria intra-hipocampal dorsal, 12) sulco colateral - cavidade do ventrículo lateral, 13) alveus, 14) superfície ventricular do hipocampo, 15) fímbria do hipocampo, 16) eminência colateral.

Os grandes vasos encontrados nas cisternas são importantes por duas razões: a) porque são responsáveis pela vascularização arterial e drenagem venosa das estruturas a serem ressecadas, b) porque devem ser preservados pela importância dos territórios que vascularizam a montante da região operada. As etapas cirúrgicas da amigdalohipocampectomia seletiva pela técnica de Niemeyer podem ser visualizadas na Figura 7.

A artéria coroidéia anterior tem origem na parede posterior da artéria carótida interna à montante da artéria comunicante posterior. Em seu curso ocupa inicialmente a cisterna carotídea e posteriormente as cisternas crural e ambiens. Após cruzar a cisterna ambiens chega à fissura coroidéia para vascularizar o plexo coróide do corno temporal. Os ramos que vascularizam o hipocampo originam-se na cisterna ambiens e na fissura coroidéia.

A artéria cerebral posterior, que se origina pela bifurcação do tronco basilar apresenta quatro seguimentos; a) denominado P1 vai da bifurcação da basi- 


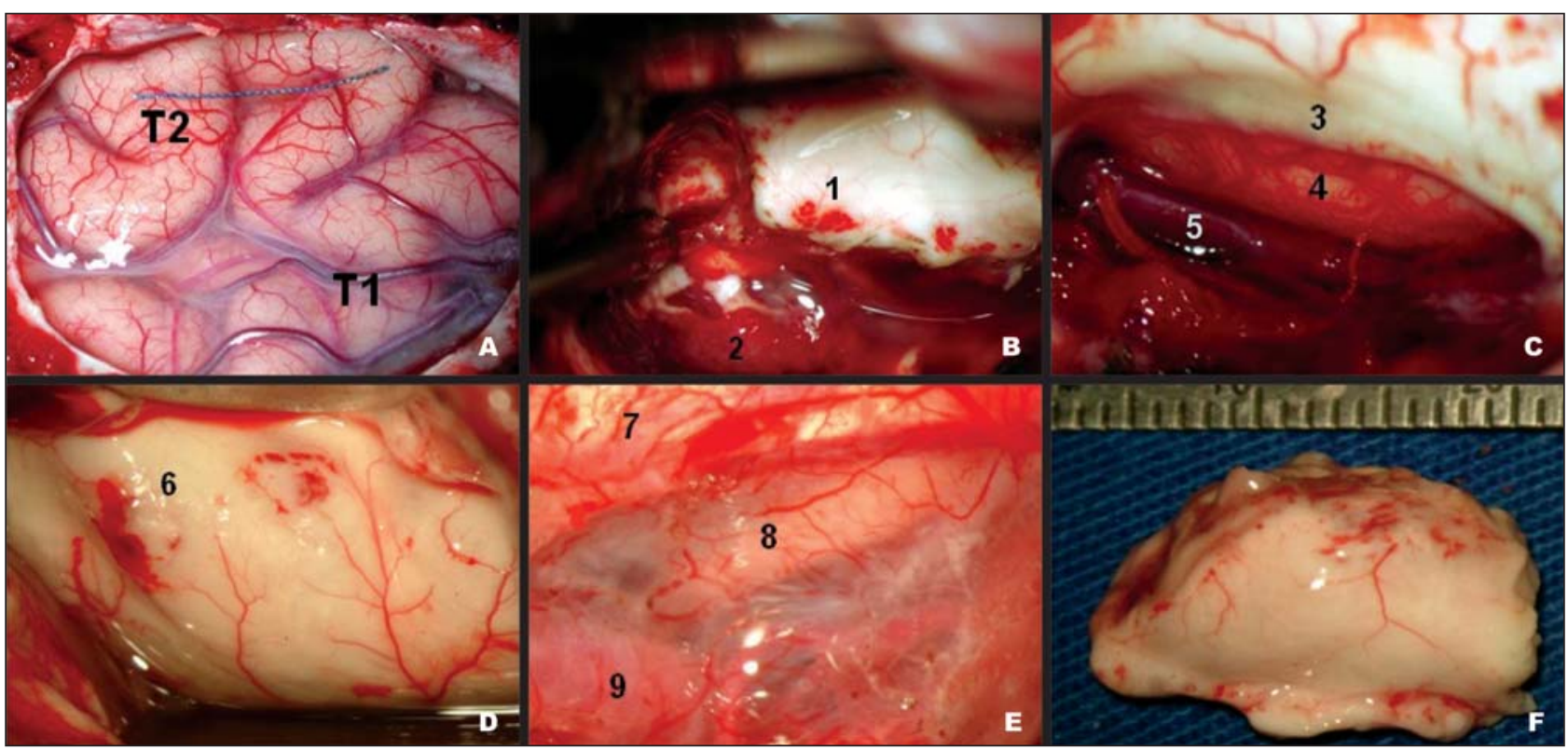

Fig 7. Etapas cirúrgicas da amigdalohipocampectomia seletiva a direita, com exceção de $A$ (lado esquerdo), pela técnica de Niemeyer. A) exposição dos giros temporal superior ( $\left.T_{1}\right)$ e médio ( $\left.T_{2}\right)$. Linha verde em $T_{2}$ evidenciando o local da corticotomia. B) a primeira etapa após entrar na cavidade ventricular é a ressecção da amígdala. C) a fissura coroidéia é aberta expondo as estruturas vasculares na cisterna ambiens. D) hipocampo de tamanho normal evidenciando as digitações. E) aspecto da membrana aracnóide preservada e visualização das estruturas das cisternas ambiens e crural por transparência. F) peça de hipocampo ressecada em bloco. 1) cabeça do hipocampo, 2) plexo coróide, 3) fímbria, 4) parahipocampo, 5) artéria cerebral posterior, 6) digitações do hipocampo, 7) tenda do cerebelo, 8) nervo oculomotor, 9) segmento $P_{2}$ da artéria cerebral posterior.

lar até a união da cerebral posterior com a comunicante posterior. Os ramos principais de $\mathrm{P}_{1}$ são as artérias talamoperfurantes e a artéria coroidéia posterior medial, 2) $\mathrm{P}_{2}$, entre a anastomose com comunicante posterior e a emergência das artérias temporais inferiores, 3) o seguimento $\mathrm{P}_{3}$ origina-se lateralmente a porção posterior do mesencéfalo penetrando na cisterna quadrigeminal. O seguimento $P_{2}$ e a porção anterior e média de $\mathrm{P}_{3}$ relacionam-se diretamente com a fissura hipocampal. Do seguimento $\mathrm{P}_{3}$ deriva a vascularização do para-hipocampo em suas porções média e posterior, 4) o seguimento $\mathrm{P}_{4}$ é representado pelo trajeto cortical da artéria na fissura inter-hemisférica e não vasculariza a região temporal mesial.

O hipocampo é vascularizado pelas artérias hipocampais que penetram pelo giro denteado, sulco fimbrio - denteado e pelo sulco hipocampal. As artérias hipocampais em número de 2 a 7, com um diâmetro entre 200 e 800 micras, dividem-se em 3 grandes troncos ${ }^{8}$ :

1. Artéria hipocampal anterior, geralmente ramo da artéria cerebral posterior das artérias temporais anteriores é responsável pela vascularização da cabeça do hipocampo. Em 29,4\% dos casos pode se originar da artéria coroidéia anterior e cursa entre o úncus hipocampal e o parahipocampo.
2. Artéria hipocampal média, originada dos segmentos $\mathrm{P}_{2}$ ou $\mathrm{P}_{3}$ da artéria cerebral posterior ou das artérias temporais inferiores, irriga o corpo do hipocampo. Recebe em muitas publicações a denominação de artéria de Uchimura ${ }^{9}$.

3. Artéria hipocampal posterior, ramo das artérias coroidéia posteriores laterais e das artérias espleniais, responsável pela vascularização da porção caudal da formação hipocampal.

O vaso dominante na vacularização da formação hipocampal é a artéria hipocampal média. Quando muito calibrosa pode substituir as artérias hipocampais anterior e posterior ${ }^{9}$. Marinkokovic ${ }^{8}$ descreve anastomoses em forma de arcos entre as artérias hipocampais anterior, média e posterior formando um anel arterial na base do sistema límbico.

No interior do hipocampo os três grandes troncos arteriais dão origem às chamadas artérias retas, que se dividem em grandes artérias hipocampais dorsais e ventrais e pequenas artérias hipocampais ventrais, mediais e dorsais. Estes vasos intrahipocampais suprem a formação hipocampal numa organização segmentar.

O subículo próprio é suprido por pequenos vasos ventrais, ramos diretos das artérias retas que emer- 
gem das artérias hipocampais. O pro-subículo é vascularizado por pequenos ramos ventrais e ocasionalmente por ramos das grandes artérias ventrais. O setor CA1 do hipocampo é suprido por grandes artérias ventrais. A porção mais dorsal deste setor é algumas vezes irrigada por uma grande artéria dorsal. Os setores $C_{2}$ e $C_{3}$ recebem vascularização de grandes artérias dorsais e de pequenos ramos dorsais enquanto que o setor $\mathrm{CA}_{4}$ é irrigado por pequenos ramos dorsais. A rede capilar destas artérias apresenta diferentes densidades dependendo da região da formação hipocampal. Os capilares apresentam uma densidade maior nas áreas com maior número de sinapses: camada piramidal e molecular do hipocampo e camada molecular do giro denteado.

As artérias intra-hipocampais podem apresentar importantes variações em seus territórios de irrigação. Em muitos casos o campo CA1 pode ser vascularizado unicamente por uma grande artéria ventral enquanto que CA2 e CA3 possuem uma abundante aferência arterial derivada da anastomose de inúmeros grupos vasculares. Esta característica especifica da vascularização de CA1 associada ao longo curso de suas artérias aferentes suportam a teoria da vulnerabilidade desta estrutura aos eventos isquêmicos ${ }^{10,11}$.

Medidas aferidas na RNM pré-operatória - As distâncias e os respectivos desvios-padrões das linhas $A$, $B$ e $C$ foram, respectivamente, no lado direito 2,14; 3,0 , and $3,4 \mathrm{~cm}$. As mesmas medidas no lado esquerdo foram 2,18; 2,95 e $3,7 \mathrm{~cm}$.

\section{DISCUSSÃO}

A amígdalo-hipocampectomia seletiva e a lobectomia temporal anterior são as duas técnicas mais utilizadas no tratamento da epilepsia do lobo temporal. A lobectomia temporal anterior standard envolve ressecção de $4.5 \mathrm{~cm}$ anteriores dos giros temporais médio e inferior, sendo que a porção inferior do giro temporal superior pode ser incluída. No que diz respeito à amígdalo-hipocampectomia seletiva, duas vias classicamente descritas podem ser utilizadas: a transilviana e a transcortical. A via transcortical pode ser realizada não somente através do giro temporal médio, como proposto por Niemeyer, mas também através da parte anterior do giro temporal superior ${ }^{12}$, do sulco temporal superior ${ }^{13}$ ou subtemporal ${ }^{14}$. O objetivo da amígdalo-hipocampectomia seletiva é ressecar a parte anterior do hipocampo (1,5 a 2,5 cm, ou mais), parte do parahipocampo, o giro dentato, o úncus e a parte temporal da amígdala. A ressecção pode ser extendida lateralmente baseado nos estudos eletro- diagnósticos, lado da dominância da fala, anormalidades neocorticais ou monitorização invasiva. Quando a patologia e o tipo de crise são restritos a região temporal mesiobasal, procedimento seletivo deve ser realizado. A via transilviana postulada por Yasargil, por sua vez, tem como principal vantagem a preservação das radiações ópticas ${ }^{15-18}$, o que é comprovado por vários estudos anatômicos ${ }^{19-23}$. Por outro lado, embora anatomicamente as radiações ópticas sejam mais lesadas quando a via transcortical é utilizada, os pacientes geralmente não relatam sintomas visuais no pós-operatório. Estudos prospectivos comparando as duas técnicas e utilizando avaliações mais objetivas como campimetria pré e pós-operatória, por exemplo, são necessários para alicerçar condutas com alto grau de evidência clínico-epidemiológica.

No que diz respeito à vascularização do hipocampo, está bem conhecido na literatura o conceito de diferentes comportamentos frente à isquemia dos diferentes setores do hipocampo. As porções mais sensíveis seriam $C_{1}$ 1, também chamado setor de Sommer, e $\mathrm{CA}_{4}$ (endofolium), enquanto que, $\mathrm{CA}_{2}$ e $\mathrm{CA}_{3}$, denominado setor de Spielmeyer, demonstra resistência aos eventos isquêmicos ${ }^{24}$. Tal realidade pode estar fundamentada nas variações dos vasos intra-hipocampais anteriormente descritas. Ademais, CA1 apresentaria um número menor de capilares em relação às demais partes do hipocampo além de possuir neurônios piramidais com características metabólicas e receptores de membrana mais sensíveis à isquemia.

Nas últimas décadas, no entanto, as teorias que apontam os eventos isquêmicos como causa das lesões hipocampais, vem sendo contestadas por vários auto$\operatorname{res}^{25-27}$, que defendem a hipótese de que o substrato das lesões residem nas características metabólicas dos neurônios, nas sinapses e especialmente nos receptores dos neurônios piramidais. Estas observações suportam o conceito de que a estrutura celular especifica dos diversos setores do hipocampo são as responsáveis pelas alterações anatômicas observadas na EH.

No que concerne às medidas pré-operatórias utilizadas para maximizar a exposição cirúrgica da amígdala e do hipocampo, evitando ampliação da corticotomia e retração cerebral, os autores consideram que a mensuração dos parâmetros anatômicos estudados na RNM pode auxiliar o cirurgião no transoperatório, pois as medidas são individualizadas para cada caso e as eventuais alterações na anatomia, tais como tamanho do hipocampo, do corno temporal do ventrículo lateral, distância do córtex temporal ou extremidade anterior do lobo temporal ao corno temporal, são mensuradas previamente à cirurgia. 
Em conclusão, o conhecimento da anatomia microcirúrgica do lobo temporal pode ser compreendido de forma tridimensional quando diferentes perspectivas anatômicas analisam uma mesma área e suas correlações adjacentes. Como a anatomia hipocampal verificada na cirúrgica comumente apresenta variações no volume do hipocampo a ser ressecado, as medidas pré-operatórias adquiridas na RNM podem ser guias anatômicos úteis na corticotomia na técnica de Niemeyer.

Agradecimentos - Um dos autores (GRI) é especialmente grato aos professores Evandro de Oliveira durante período de research fellowship no Instituto de Ciências Neurológicas de São Paulo (Laboratório de Microcirurgia), Ossama Al-Mefty e M. Gazi Yasargil, durante período de research fellowship na University of Arkansas for Medical Sciences.

\section{REFERENCES}

1. Wen HT, Rhoton AL Jr, Oliveira E, et al. Microsurgical anatomy of the temporal lobe: Part 1: Mesial temporal lobe anatomy and its vascular relationships as applied to amygdalohippocampectomy. Neurosurgery 1999;45:549-592.

2. Duvernoy HM. The human brain, surface, blood supply, and three-dimensional sectional anatomy. 2.Ed. Vienna: Springer-Verlag, 1999:122-143.

3. Gloor P: The temporal lobe and limbic system. New York: Oxford University Press, 1997.

4. Paglioli E, Cendes F. Epilepsia: tratamento cirúrgico. In Guerreiro CAM, Guerreiro MM, Cendes F, Lopes-Cendes I (org). 3.Ed. Epilepsia, 2000.

5. Niemeyer P. The transventricular amygdala-hippocampectomy in temporal lobe epilepsy. In Baldwin M, Bailey $\mathrm{P}$ (Eds). The temporal lobe epilepsy. Springfield, IL: Charles C Thomas, 1958:461-482.

6. Klinger J. Die makroskopische Anatomie der Ammons-formation Denkschriften der schweizerischen naturforschenden Gesellschaft. Zurich: Fretz, 1948;78:82

7. Yasargil MG. Microneurosurgery. Stuttgart: Georg Thieme Verlag, 1984-1996

8. Marinkokovic S, Miliasavljevic S, Puskas L. Microvascular anatomy of the hippocampal formation: Surg Neurol 1992;37:339-349.
9. Uchimura J. Uber die Gefassversorgung des Ammonshornes. Z Ges Neurol Psychiatr 1928:112:1-19.

10. Scharrer E. Vascularization and vulnerability of the cornu ammonis in the opossum. Arch Neurol Psychiatry 1940;44:483-506.

11. Gastaut H, Lamemrs JH. Anatomie du rhinencephale. Paris: Masson; 1961.

12. Olivier A. Transcortical selective amygdalohippocampectomy in temporal lobe epilepsy. Can J Neurol Sci 2000;27(Suppl 1):S68-S76.

13. Rougier A, Saint-Hilaire J, Loiseau P, et al. Evaluation and surgical treatment of the epilepsies. Neurochirurgie 1992;38:3-112.

14. Hori T, Tabuchi S, Kurosaki M, et al. Subtemporal amygdalohippocampectomy for treating medically intractable temporal lobe epilepsy. Neurosurgery 1993;33:50-57.

15. Yasargil MG, Cravens GF, Roth P. Surgical approaches to "inaccessible" brain tumors. Clin Neurosurg 1988;34:42-110.

16. Yasargil MG, Teddy PJ, Roth P. Selective amygdalo-hippocampectomy: operative anatomy and surgical technique. Adv Tech Stand Neurosurg 1985; 12:93-123

17. Yasargil MG, Wieser HG, Valavanis A, et al. Surgery and results of selective amygdala-hippocampectomy in one hundred patients with nonlesional limbic epilepsy. Neurosurg Clin N Am 1993;4:243-261.

18. Yasargil MG, Wieser HG: Selective amygdalohippocampectomy at the University Hospital, Zurich. In Engel J (Ed). Surgical treatment of the epilepsies. New York: Raven, 1987:653-658.

19. Türe U, Yasargil MG, Friedman AH, et al. Fiber dissection technique: lateral aspect of the brain. Neurosurgery 2000;47:417-427.

20. Türe U, Yasargil MG, Pait TG. Is there a superior occipitofrontal fasciculus? A microsurgical anatomic study. Neurosurgery 1997;40:1226-1232.

21. Rubino PA, Rhoton AL Jr, Tong X, Oliveira E. Three-dimensional relationships of the optic radiation. Neurosurgery 2005;(Suppl):S219-S227.

22. Castro I, Christoph DH, Santos DP, Landeiro JA. Internal structure of the cerebral hemispheres: an introduction of fiber dissection technique. Arq Neuropsiquiatr 2005;63:252-258.

23. Sincoff EH, Tan Y, Abdulrauf SI. White fiber dissection of the optic radiations of the temporal lobe and implications for surgical approaches to temporal horn. J Neurosurg 2004;101:739-746.

24. Spielmeyer W. The anatomic substratum of convulsive state. Arch Neurol Psychiatry 1930;23:869-875.

25. Margerison JH, Corsellis JAN. Epilepsy and temporal lobes. Brain 1966;89:499-530.

26. Fried RL. The histochemical architecture of the Ammon's horn as related to its selective vulnerability. Acta Neuropathol 1966;6:1-13.

27. De Reuck J, De Koster W. Ischemimic lesions of the hippocampus and their relation to Amon's horn sclerosis. J Neurol 1979;220:157-168. 